\title{
Common New Fixed Point Theorem in Modified Intuitionistic Fuzzy Metric Spaces Using Implicit Relation
}

\author{
Saurabh Manro ${ }^{1}$, Sumitra ${ }^{2}$ \\ ${ }^{1}$ School of Mathematics and Computer Applications, Thapar University, Patiala, India \\ ${ }^{2}$ Department of Mathematics, Faculty of Science, Jazan University, Jazan, KSA \\ Email: sauravmanro@yahoo.com, sauravmanro@hotmail.com,mathsqueen_d@yahoo.com
}

Received May 5, 2013; revised June 5, 2013; accepted June 13, 2013

Copyright (C) 2013 Saurabh Manro, Sumitra. This is an open access article distributed under the Creative Commons Attribution License, which permits unrestricted use, distribution, and reproduction in any medium, provided the original work is properly cited.

\begin{abstract}
In this paper, we prove some common fixed point theorems for two pair of compatible and subsequentially continuous mappings satisfying an implicit relation in Modified Intuitionistic fuzzy metric spaces. Consequently, our results improve and sharpen many known common fixed point theorems available in the existing literature of metric fixed point theory.
\end{abstract}

Keywords: Modified Intuitionistic Fuzzy Metric Space; Compatible Mappings; Subcompatible Mappings;

Subsequential Continuous Mappings; Reciprocal Continuity; Implicit Relation

\section{Introduction}

The concept of fuzzy set was introduced in 1965 by Zadeh [1]. In 1986, with similar endeavour, Atanasov [2] introduced and studied the concept of Intuitionistic fuzzy sets (IFS). Using the idea of IFS, a generalization of fuzzy metric space was introduced by Park [3] which is known as Intuitionistic fuzzy metric space. Since the Intuitionistic fuzzy metric space has extra conditions (see [2]), Saadati et al. [4] reframed the idea of Intuitionistic fuzzy metric space and proposed a new notion under the name of Modified Intuitionistic fuzzy metric space by introducing idea of continuous $t$-representable.

In 1986, Jungck [5] introduced the notion of compatible maps for a pair of self mappings. Jungck et al. [6] initiated the study of weakly compatible maps in metric space. With a view to improve commutativity conditions in common fixed point theorems, Sessa [7] introduced the notion of weakly commuting pair. Most recently, Bouhadjera et al. [8] (see also [9]) introduced two new notions namely: subsequential continuity and subcompatibility.

In this paper, we prove some common fixed point theorems for two pair of compatible and subsequentially continuous mappings satisfying an implicit relation in modified Intuitionistic fuzzy metric spaces. Consequently, our results improve and sharpen many known com- mon fixed point theorems available in the existing literature of metric fixed point theory and generalize the results of D. Gopal et al. [10, Theorem 3.1 and Theorem 3.2].

\section{Preliminaries}

Lemma 2.1. [11] Consider the set $L^{*}$ and the operation $\leq_{L^{*}}$ defined by

$$
\begin{aligned}
& L^{*}=\left\{\left(x_{1}, x_{2}\right):\left(x_{1}, x_{2}\right) \in[0,1]^{2}, x_{1}+x_{2} \leq 1\right\} \\
& \left(x_{1}, x_{2}\right) \leq_{L^{*}}\left(y_{1}, y_{2}\right) \Leftrightarrow x_{1} \leq y_{1}, x_{2} \geq y_{2},
\end{aligned}
$$

for every $\left(x_{1}, x_{2}\right),\left(y_{1}, y_{2}\right) \in L^{*}$. Then $\left(L^{*}, \leq_{L^{*}}\right)$ is a complete lattice.

We denote its units by $0_{L^{*}}=(0,1)$ and $1_{L^{*}}=(1,0)$.

Definition 2.1. [12] A triangular norm (t-norm) on $L^{*}$ is a mapping $\mathscr{T}:\left(L^{*}\right)^{2} \rightarrow L^{*}$ satisfying the following conditions:

(1) $\mathscr{T}\left(x, 1_{L^{*}}\right)=x$ for all $x \in L^{*}$,

(2) $\mathscr{T}(x, y)=\mathscr{T}(y, x)$ for all $x, y \in L^{*}$,

(3) $\mathscr{T}(x, \mathscr{F}(y, z))=\mathscr{F}(\mathscr{F}(x, y), z)$ for all $x, y, z$ $\in L^{*}$,

(4) If for all $x, x^{\prime}, y, y^{\prime} \in L^{*}, x \leq_{L^{*}} x^{\prime}$ and $y \leq_{L^{*}} y^{\prime}$ implies

$$
\mathscr{F}(x, y) \leq_{L^{*}} \mathscr{F}\left(x^{\prime}, y^{\prime}\right) .
$$


Definition 2.2. $[11,12]$ A continuous $t$-norm $\mathrm{F}$ on $L^{*}$ is called continuous $t$-representable iff there exist a continuous $t$-norm * and a continuous $t$-conorm $\diamond$ on $[0,1]$ such that for all $x=\left(x_{1}, x_{2}\right), y=\left(y_{1}, y_{2}\right) \in L^{*}$,

$\mathscr{F}(x, y)=\left(x_{1} * y_{1}, x_{2} \diamond y_{2}\right)$.

Definition 2.3. [4] Let $M, N$ are fuzzy sets from $X^{2} \times(0,+\infty) \rightarrow[0,1]$ such that

$M(x, y, t)+N(x, y, t) \leq 1$ for all $x, y \in X$ and $t>0$. The 3-tuple $\left(X, \zeta_{M, N}, \mathscr{F}\right)$ is said to be a Modified Intuitionistic fuzzy metric space if $X$ is an arbitrary non empty set, $\mathscr{F}$ is a continuous $t$-representable and $\zeta_{M, N}$ is a mapping $X^{2} \times(0,+\infty) \rightarrow L^{*}$ satisfying the following conditions for every $x, y \in X$ and $t, s>0$ :
(a) $\zeta_{M, N}(x, y, t)>_{L^{*}} 0_{L^{*}}$;
(b) $\zeta_{M, N}(x, y, t)=1_{L^{*}}$ iff $x=y$;
(c) $\zeta_{M, N}(x, y, t)=\zeta_{M, N}(y, x, t)$;
$\zeta_{M, N}(x, y, t+s)$
$\geq_{L^{*}} \mathscr{F}\left(\zeta_{M, N}(x, z, t), \zeta_{M, N}(z, y, s)\right)$;
(e) $\zeta_{M, N}(x, y,):.(0,+\infty) \rightarrow L^{*}$ is continuous.

In this case, $\zeta_{M, N}$ is called an Intuitionistic fuzzy metric. Here,

$$
\zeta_{M, N}(x, y, t)=(M(x, y, t), N(x, y, t)) .
$$

In the sequel, we will call $\left(X, \zeta_{M, N}, \mathscr{F}\right)$ to be just a Modified Intuitionistic fuzzy metric space.

Remark 2.1. [13] In Modified Intuitionistic fuzzy metric space $\left(X, \zeta_{M, N}, \mathcal{F}\right), M(x, y,$.$) is non decreasing$ and $N(x, y,$.$) is non-increasing for all x, y \in X$. Hence $\zeta_{M, N}(x, y, t)$ is non-decreasing with respect to $t$ for all $x, y \in X$.

Definition 2.4. [4] A sequence $\left\{x_{n}\right\}$ in a Modified Intuitionistic fuzzy metric space $\left(X, \zeta_{M, N}, \mathscr{F}\right)$ is called a Cauchy sequence if for each $\varepsilon>0$ and $t>0$, there exists $n_{0} \in \mathbb{N}$ such that $\zeta_{M, N}\left(x_{n}, x_{m}, t\right)>_{L^{*}}(1-\varepsilon, \varepsilon)$ for each $n, m \geq n_{0}$ and for all $t$.

Definition 2.5. [4] A sequence $\left\{x_{n}\right\}$ in a Modified Intuitionistic fuzzy metric $\operatorname{spac}\left(X, \zeta_{M, N}, \mathscr{F}\right)$ is said to be convergent to $x \in X$, denoted by $x_{n} \rightarrow X$ if $\lim _{n \rightarrow \infty} \zeta_{M, N}\left(x_{n}, x, t\right)=1_{L^{*}}$ for all $t$.

A Modified Intuitionistic fuzzy metric space $\left(X, \zeta_{M, N}, \mathscr{F}\right)$ is said to be complete iff every Cauchy sequence is converges to a point of it.

Definition 2.6. [14] Let $f$ and $g$ be maps from a Modified Intuitionistic fuzzy metric space $\left(X, \zeta_{M, N}, \mathscr{F}\right)$ into itself. The maps $f$ and $g$ are said to be weakly commuting if $\zeta_{M, N}(A S z, S A z, t) \geq_{L^{*}} \zeta_{M, N}(A z, S z, t)$ for all $z \in X$ and $t>0$.

Definition 2.7. [4] A pair of self mappings $(f, g)$ of Modified Intuitionistic fuzzy metric space $\left(X, \zeta_{M, N}, \mathscr{F}\right)$ is said to be compatible if $\lim _{n \rightarrow \infty} \zeta_{M, N}\left(f g x_{n}, g f x_{n}, t\right)=1_{L^{*}}$ whenever $\left\{x_{n}\right\}$ is a sequence in $X$ such that $\lim _{n \rightarrow \infty} f x_{n}=\lim _{n \rightarrow \infty} g x_{n}=z$ for some $z \in X$.

Definition 2.8. [13] Two self-mappings $f$ and $g$ are called non-compatible if there exists at least one sequence $\left\{x_{n}\right\}$ such that $\lim _{n \rightarrow \infty} f x_{n}=\lim _{n \rightarrow \infty} g x_{n}=z$ for some $z \in X$ but either $\lim _{n \rightarrow \infty} \zeta_{M, N}\left(f g x_{n}, g f x_{n}, t\right) \neq 1_{L^{*}}$ or the limit does not exist for all $z \in X$.

Definition 2.9. [15] A pair of self mappings $(f, g)$ of Modified Intuitionistic fuzzy metric space $\left(X, \zeta_{M, N}, \mathscr{F}\right)$ is said to be weakly compatible if they commute at coincidence points i.e. if $f u=g u$ for some $u$ $\in X$, then $f g u=g f u$.

Definition 2.10. [16] A pair of self mappings $(f, g)$ of Modified Intuitionistic fuzzy metric space

$\left(X, \zeta_{M, N}, \mathscr{F}\right)$ is said to be occasionally weakly compatible (owc) if the pair $(f, g)$ commutes at least one coincidence point i.e. there exists at least one point $x \in X$ such that $f x=g x$ and $f g x=g f x$.

Definition 2.11. [9] Let $f$ and $g$ be maps from a Modified Intuitionistic fuzzy metric space $\left(X, \zeta_{M, N}, \mathscr{F}\right)$ into itself. The maps $f$ and $g$ are said to be subcompatible if there exist a sequence $\left\{x_{n}\right\}$ in $X$ with $\lim _{n \rightarrow \infty} f x_{n}=\lim _{n \rightarrow \infty} g x_{n}=z$ for $z \in X$ and for all $t>0$, $\lim _{n \rightarrow \infty} \zeta_{M, N}\left(A S x_{n}, S A x_{n}, t\right)=1_{L^{*}}$.

Definition 2.12. [10] Let $f$ and $g$ be maps from a Modified Intuitionistic fuzzy metric space $\left(X, \zeta_{M, N}, \mathscr{F}\right)$ into itself. The maps $f$ and $g$ are said to be reciprocally continuous if for a sequence $\left\{x_{n}\right\}$ in $X$ $\lim _{n \rightarrow \infty} f g x_{n}=f z, \lim _{n \rightarrow \infty} g f x_{n}=g z$, whenever $\lim _{n \rightarrow \infty} f x_{n}=$ $\lim _{n \rightarrow \infty} g x_{n}=z$ for some $z \in X$ and for all $t>0$.

Definition 2.13. [17] Let $f$ and $g$ be two maps from modified intuitionistic fuzzy metric space $\left(X, \zeta_{M, N}, F\right)$ into itself. The maps $f$ and $g$ are said to be subsequentially continuous if there exist a sequence $\left\{x_{n}\right\}$ in $X$ such that

$$
\lim _{n \rightarrow \infty} f x_{n}=\lim _{n \rightarrow \infty} g x_{n}=z \text { for some } z \in X
$$

and $\lim _{n \rightarrow \infty} f g x_{n}=f z, \lim _{n \rightarrow \infty} g f x_{n}=g z$ for all $t>0$.

\section{Main Results}

Implicit relations play important role in establishing of common fixed point results.

Let $\mathrm{M}_{6}$ be the set of all continuous functions

$\phi:\left(L^{*}\right)^{6} \rightarrow L^{*}$ satisfying the following conditions (for all 
$u, v \in L^{*}, u=\left(u_{1}, u_{2}\right), v=\left(v_{1}, v_{2}\right)$ and $\left.1_{L^{*}}=(1,0), 0_{L^{*}}=(0,1)\right)$ :

(A) $\phi\left(u, 1_{L^{*}}, 1_{L^{*}}, u, u, 1_{L^{*}}\right)<_{L^{*}} 0_{L^{*}}$ and

(B) $\phi\left(u, 1_{L^{*}}, u, 1_{L^{*}}, 1_{L^{*}}, u\right)<_{L^{*}} 0_{L^{*}}$

for all $u>_{L^{*}} 0_{L^{*}}$.

Example 3.1. Define $\phi:\left(L^{*}\right)^{6} \rightarrow L^{*}$ as $\phi\left(t_{1}, t_{2}, t_{3}, t_{4}, t_{5}, t_{6}\right)=t_{1}-\phi_{1}\left(\min \left\{t_{2}, t_{3}, t_{4}, t_{5}, t_{6}\right\}\right)$, where $\phi_{1}: L^{*} \rightarrow L^{*}$ is increasing and continuous function such that $\phi_{1}(s)>_{L^{*}} s$ for all $s \in(0,1)$. Clearly, $\phi$ in $\mathrm{M}_{6}$. We begin with following observation:

Theorem 3.1. Let $A, B, S$ and $T$ be four self mappings of a Modified Intuitionistic fuzzy metric space $\left(X, \zeta_{M, N}, \mathscr{F}\right)$. If the pairs $(A, S)$ and $(B, T)$ are compatible and subsequentially continuous mappings, then

(3.1) the pair $(A, S)$ has a coincidence point,

(3.2) the pair $(B, T)$ has a coincidence point.

Further, $A, B, S$ and $T$ have a unique common fixed point provided $A, B, S$ and $T$ satisfy the following:

(3.3) for any $x, y \in X, \phi$ in $M_{6}$ and for all $t>0$,

$$
\begin{aligned}
& \phi\left(\zeta_{M, N}(A x, B y, t), \zeta_{M, N}(S x, T y, t),\right. \\
& \quad \zeta_{M, N}(S x, A x, t), \zeta_{M, N}(T y, B y, t), \\
& \left.\quad \zeta_{M, N}(S x, B y, t), \zeta_{M, N}(T y, A x, t)\right) \geq_{L^{*}} 0_{L^{*}}
\end{aligned}
$$

Proof. Since the pairs $(A, S)$ and $(B, T)$ are compatible and subsequentially continuous mappings, therefore there exist sequences $\left\{x_{n}\right\}$ and $\left\{y_{n}\right\}$ in $X$ such that

$\lim _{n \rightarrow \infty} A x_{n}=\lim _{n \rightarrow \infty} S x_{n}=z$,

$\lim _{n \rightarrow \infty} B y_{n}=\lim _{n \rightarrow \infty} T y_{n}=w$ for some $z, w \in X$ and

$\lim _{n \rightarrow \infty} \zeta_{M, N}\left(A S x_{n}, S A x_{n}, t\right)=\zeta_{M, N}(A z, S z, t)=1_{L^{*}}$

and $\lim _{n \rightarrow \infty} \zeta_{M, N}\left(B T x_{n}, T B x_{n}, t\right)=\zeta_{M, N}(B w, T w, t)=1_{L^{*}}$

so that $A z=S z$ and $B w=T w$ i.e. $z$ is a coincidence point of $A$ and $S$ where as $w$ is a coincidence point of $B$ and $T$, which proves (3.1) and (3.2).

Now, we prove that $z=w$, if not, then by using (3.3), we have

$$
\begin{aligned}
& \phi\left(\zeta_{M, N}\left(A x_{n}, B y_{n}, t\right), \zeta_{M, N}\left(S x_{n}, T y_{n}, t\right)\right. \\
& \quad \zeta_{M, N}\left(S x_{n}, A x_{n}, t\right), \zeta_{M, N}\left(T y_{n}, B y_{n}, t\right), \\
& \left.\quad \zeta_{M, N}\left(S x_{n}, B y_{n}, t\right), \zeta_{M, N}\left(T y_{n}, A x_{n}, t\right)\right) \geq_{L^{*}} 0_{L^{*}}
\end{aligned}
$$

which on making $n \rightarrow \infty$ reduces to

$$
\begin{aligned}
& \phi\left(\zeta_{M, N}(z, w, t), \zeta_{M, N}(z, z, t),\right. \\
& \zeta_{M, N}(z, z, t), \zeta_{M, N}(z, w, t), \\
& \left.\zeta_{M, N}(z, w, t), \zeta_{M, N}(z, z, t)\right) \geq_{L^{*}} 0_{L^{*}} \\
& \phi\left(\zeta_{M, N}(z, w, t), 1_{L^{*}}, 1_{L^{*}}, \zeta_{M, N}(z, w, t),\right. \\
& \left.\quad \zeta_{M, N}(z, w, t), 1_{L^{*}}\right) \geq_{L^{*}} 0_{L^{*}}
\end{aligned}
$$

a contradiction to (A) so that $z=w$.

Now, we assert that $A z=z$, if not, then by (3.3), we get

$$
\begin{aligned}
& \phi\left(\zeta_{M, N}\left(A z, B y_{n}, t\right), \zeta_{M, N}\left(S z, T y_{n}, t\right),\right. \\
& \quad \zeta_{M, N}(S z, A z, t), \zeta_{M, N}\left(T y_{n}, B y_{n}, t\right), \\
& \left.\quad \zeta_{M, N}\left(S z, B y_{n}, t\right), \zeta_{M, N}\left(T y_{n}, A z, t\right)\right) \geq_{L^{*}} 0_{L^{*}}
\end{aligned}
$$

taking the limit as $n \rightarrow \infty$, we get

$$
\begin{gathered}
\phi\left(\zeta_{M, N}(A z, z, t), \zeta_{M, N}(z, z, t),\right. \\
\zeta_{M, N}(z, A z, t), \zeta_{M, N}(z, z, t), \\
\left.\zeta_{M, N}(z, z, t), \zeta_{M, N}(z, A z, t)\right) \geq_{L^{*}} 0_{L^{*}} \\
\phi\left(\zeta_{M, N}(A u, z, t), 1_{L^{*}}, \zeta_{M, N}(A u, z, t), 1_{L^{*}},\right. \\
\left.1_{L^{*}, \zeta_{M, N}}(A u, z, t)\right) \geq_{L^{*}} 0_{L^{*}}
\end{gathered}
$$

which is a contradiction to (B). Therefore, $A z=z=S z$.

Similarly, we prove that $B z=z=T z$ by using (3.3). Therefore, in all, $z=A z=B z=S z=T z$. i.e. $z$ is common fixed point of $A, B, S$ and $T$. The uniqueness of common fixed point is an easy consequence of the inequality (3.3). This completes the proof of the theorem. $\square$

Theorem 3.2. Let $A, B, S$ and $T$ be four self mappings of a Modified Intuitionistic fuzzy metric space $\left(X, \zeta_{M, N}, \widetilde{F}\right)$. If the pairs $(A, S)$ and $(B, T)$ are subcompatible and reciprocally continuous mappings, then

(3.4) the pair $(A, S)$ has a coincidence point,

(3.5) the pair $(B, T)$ has a coincidence point.

Further, $A, B, S$ and $T$ have a unique common fixed point provided $A, B, S$ and $T$ satisfy the condition (3.3).

Proof. Proof easily follows on same lines of Theorem 3.1 and using definition of reciprocally continuous and subcompatible mappings.

Corollary 3.1. The conclusions of Theorem 3.1 and Theorem 3.2 remain true if we replace the inequality (3.3) by any one of the following:

$$
\begin{aligned}
& \zeta_{M, N}(A x, B y, t) \\
& \geq_{L^{*}} F( \min \left\{\zeta_{M, N}(S x, T y, t), \zeta_{M, N}(S x, A x, t),\right. \\
& \zeta_{M, N}(T y, B y, t), \zeta_{M, N}(S x, B y, t), \\
&\left.\left.\zeta_{M, N}(T y, A x, t)\right\}\right) ;
\end{aligned}
$$

where $F:[0,1] \rightarrow[0,1]$ is increasing and continuous function such that $F(t)>_{L^{*}} t$ for all $t \in(0,1)$.

$$
\int_{0}^{\zeta_{M, N}(A x, B y, t)} \phi(t) d t \geq_{L^{*}} F\left(\int_{0}^{\Omega} \phi(t) d t\right)
$$

where

$$
\Omega=\min \left\{\begin{array}{l}
\zeta_{M, N}(S x, T y, t), \zeta_{M, N}(S x, A x, t), \\
\zeta_{M, N}(T y, B y, t), \zeta_{M, N}(S x, B y, t), \\
\zeta_{M, N}(T y, A x, t)
\end{array}\right\}
$$


where $F: L^{*} \rightarrow L^{*}$ is increasing and continuous function such that $F(t)>_{L^{*}} t$ for all $t \in(0,1)$ and $\phi: \mathfrak{R}^{+} \rightarrow \mathfrak{R}^{+}$is a Lebesgue integrable function which is summable and satisfies $0<\int_{0}^{\varepsilon} \phi(s) \mathrm{d} s<1$, for all $0<\varepsilon<1$.

By setting $A=B$ in Theorems 3.1, 3.2, we derive the following corollaries for three mappings.

Corollary 3.2. Let $A, S$ and $T$ be three self mappings of a Modified Intuitionistic fuzzy metric space $\left(X, \zeta_{M, N}, \mathscr{F}\right)$. If the pairs $(A, S)$ and $(A, T)$ are compatible and subsequentially continuous mappings, then

(3.8) the pair $(A, S)$ has a coincidence point,

(3.9) the pair $(A, T)$ has a coincidence point.

Further, $A, B, S$ and $T$ have a unique common fixed point provided $A, B, S$ and $T$ satisfy the following:

(3.10) for any $x, y \in X, \phi$ in $\mathrm{M}_{6}$ and for all $\mathrm{t}>0$,

$$
\begin{aligned}
& \phi\left(\zeta_{M, N}(A x, A y, t), \zeta_{M, N}(S x, T y, t)\right. \\
& \quad \zeta_{M, N}(S x, A x, t), \zeta_{M, N}(T y, A y, t), \\
& \left.\quad \zeta_{M, N}(S x, A y, t), \zeta_{M, N}(T y, A x, t)\right) \geq_{L^{*}} 0_{L^{*}}
\end{aligned}
$$

Corollary 3.3. Let $A, S$ and $T$ be three self mappings of a Modified Intuitionistic fuzzy metric space $\left(X, \zeta_{M, N}, \mathscr{F}\right)$. If the pairs $(A, S)$ and $(A, T)$ are subcompatible and reciprocally continuous mappings, then (3.8) and (3.9) satisfied. Further, $A, S$ and $T$ have a unique common fixed point provided $A, S$ and $T$ satisfy the condition (3.10).

Alternatively, by setting $S=T$ in Theorems 3.1, 3.2, we derive the following corollaries for three mappings.

Corollary 3.4. Let $A, B$ and $S$ be three self mappings of a Modified Intuitionistic fuzzy metric space $\left(X, \zeta_{M, N}, \mathscr{F}\right)$. If the pairs $(A, S)$ and $(B, S)$ are compatible and subsequentially continuous mappings, then

(3.11) the pair $(A, S)$ has a coincidence point,

(3.12) the pair $(B, S)$ has a coincidence point.

Further, $A, B$ and $S$ have a unique common fixed point provided $A, B$ and $S$ satisfy the following:

(3.13) for any $x, y \in X, \phi$ in $\mathrm{M}_{6}$ and for all $t>0$,

$$
\begin{aligned}
& \phi\left(\zeta_{M, N}(A x, B y, t), \zeta_{M, N}(S x, S y, t),\right. \\
& \quad \zeta_{M, N}(S x, A x, t), \zeta_{M, N}(S y, B y, t), \\
& \left.\quad \zeta_{M, N}(S x, B y, t), \zeta_{M, N}(S y, A x, t)\right) \geq_{L^{*}} 0_{L^{*}}
\end{aligned}
$$

Corollary 3.5. Let $A, B$ and $S$ be three self mappings of a Modified Intuitionistic fuzzy metric space $\left(X, \zeta_{M, N}, \mathscr{F}\right)$. If the pairs $(A, S)$ and $(B, S)$ are subcompatible and reciprocally continuous mappings, then (3.11) and (3.12) satisfied. Further, $A, B$ and $S$ have a unique common fixed point provided $A, B$ and $S$ satisfy the condition (3.13).

Finally, by setting $A=B$ and $S=T$ in Theorems 3.1 and 3.2, we derive the following corollaries:
Corollary 3.6. Let $A$ and $S$ be four self mappings of a modified intuitionistic fuzzy metric space $\left(X, \zeta_{M, N}, \widetilde{F}\right)$. If the pair $(A, S)$ is compatible and subsequentially continuous mappings, then

(3.14) the pair $(A, S)$ has a coincidence point.

Further, $A$ and $S$ have a unique common fixed point provided $A$ and $S$ satisfy the following:

(3.15) for any $x, y \in X, \phi$ and $\psi$ in $\mathrm{M}_{6}$ and for all $t>0$,

$$
\begin{aligned}
& \phi\left(\zeta_{M, N}(A x, A y, t), \zeta_{M, N}(S x, S y, t),\right. \\
& \quad \zeta_{M, N}(S x, A x, t), \zeta_{M, N}(S y, A y, t), \\
& \left.\quad \zeta_{M, N}(S x, A y, t), \zeta_{M, N}(S y, A x, t)\right) \geq_{L^{*}} 0_{L^{*}}
\end{aligned}
$$

Corollary 3.7. Let $A$ and $S$ be pair of self mappings of a modified intuitionistic fuzzy metric space

$\left(X, \zeta_{M, N}, \mathscr{F}\right)$. If the pair $(A, S)$ is subcompatible and reciprocally continuous mappings, then (3.14) is satisfied. Further, $A$ and $S$ have a unique common fixed point provided $A$ and $S$ satisfy the condition (3.15).

\section{Example 3.1. [10]}

Let $\left(X, \zeta_{M, N}, \mathscr{F}\right)$ be a Modified Intuitionistic fuzzy metric space

where $X=(-3, \infty)$

and define $\phi:\left(L^{*}\right)^{6} \rightarrow L^{*}$ as

$\phi\left(t_{1}, t_{2}, t_{3}, t_{4}, t_{5}, t_{6}\right)=t_{1}-\phi_{1}\left(\min \left\{t_{2}, t_{3}, t_{4}, t_{5}, t_{6}\right\}\right)$, where $\phi_{1}: L^{*} \rightarrow L^{*}$ is increasing and continuous function such that $\phi_{1}(s)>_{L^{*}} s$ for all $s \in(0,1)$. Clearly, $\phi$ satisfies all conditions (A) and (B). Define $A, B, S$ and $T$ by

$$
A x=B x= \begin{cases}0 & \text { if } x \in(-3,0) \\ \frac{x}{3} & \text { if } x \in[0,1] \\ 2 x-1 & \text { if } x \in(1, \infty)\end{cases}
$$

and $S x=T x= \begin{cases}\frac{x}{2} & \text { if } x \in(-3,1] \\ 3 x-2 & \text { if } x \in(1, \infty) .\end{cases}$

where

$\zeta_{M, N}(x, y, t)=\left(\frac{t}{t+|x-y|}, \frac{|x-y|}{t+|x-y|}\right)$ for all

$x, y \in X=(-3, \infty), t>0$. Clearly, for the sequence

$\left\{x_{n}\right\}=\left\{\frac{1}{n}\right\},(A, S)$ and $(B, T)$ are compatible as well as subsequentially continuous. Therefore, all the conditions of Theorem 3.1 are satisfied. Evidently, $z=0$ is a coincidence as well as unique common fixed point of $A, B, S$ and $T$.

Example 3.2. [10]

Let $\left(X, \zeta_{M, N}, \mathscr{F}\right)$ be a Modified Intuitionistic fuzzy 
metric space where $X=\Re$ and define $\phi:\left(L^{*}\right)^{6} \rightarrow L^{*}$ as $\phi\left(t_{1}, t_{2}, t_{3}, t_{4}, t_{5}, t_{6}\right)=t_{1}-\phi_{1}\left(\min \left\{t_{2}, t_{3}, t_{4}, t_{5}, t_{6}\right\}\right)$, where $\phi_{1}: L^{*} \rightarrow L^{*}$ is increasing and continuous function $\phi_{1}(s)=\sqrt{s}$ such that $\phi_{1}(s)>_{L^{*}} s$ for all $s \in(0,1)$.

Clearly, $\phi$ satisfies all conditions (A) and (B). Define $A, B, S$ and $T$ by

$$
\begin{aligned}
& A x=B x= \begin{cases}x+1 & \text { if } x \in(-\infty, 1) \\
2 x-1 & \text { if } x \in[1, \infty),\end{cases} \\
& S x=T x= \begin{cases}\frac{x}{2} & \text { if } x \in(-\infty, 1) \\
3 x-2 & \text { if } x \in[1, \infty) .\end{cases}
\end{aligned}
$$

and

$\zeta_{M, N}(x, y, t)=\left(\frac{t}{t+|x-y|}, \frac{|x-y|}{t+|x-y|}\right)$ for all $X=\mathfrak{R}, t$ $>0$. Clearly, for the sequence $\left\{x_{n}\right\}=\left\{1+\frac{1}{n}\right\},(A, S)$ and $(B, T)$ are subcompatible as well as reciprocally continuous. Therefore, all the conditions of Theorem 3.2 are satisfied. Evidently, $z=0$ is a coincidence as well as unique common fixed point of $A, B, S$ and $T$.

\section{Acknowledgements}

The authors thank the referee for his/her careful reading and useful suggestions of the manuscript.

\section{REFERENCES}

[1] L. A. Zadeh, "Fuzzy Sets," Information and Control, Vol. 8, No. 3, 1965, pp. 338-353. doi:10.1016/S0019-9958(65)90241-X

[2] A. T. Atanassov, "Intuitionistic Fuzzy Sets," Fuzzy Sets and Systems, Vol. 20, No. 1, 1986, pp. 87-96. doi:10.1016/S0165-0114(86)80034-3

[3] J. H. Park, "Intuitionistic Fuzzy Metric Spaces," Chaos, Solitons \& Fractals, Vol. 22, No. 5, 2004, pp. 1039-1046. doi:10.1016/i.chaos.2004.02.051

[4] R. Saadati, S. Sedghi and N. Shobhe, "Modified Intuitionistic Fuzzy Metric Spaces and Some Fixed Point Theorems," Chaos, Solitons \& Fractals, Vol. 38, No. 1, 2008, pp. 36-47. doi:10.1016/j.chaos.2006.11.008

[5] G. Jungck, "Compatible Mappings and Common Fixed Points," International Journal of Mathematics and Mathematical Sciences, Vol. 9, No. 4, 1986, pp. 771-779.
doi:10.1155/S0161171286000935

[6] G. Jungck and B. E. Rhoades, "Fixed Points for Set Valued Functions without Continuity," Indian Journal of Pure and Applied Mathematics, Vol. 29, No. 3, 1998, pp. 227-238.

[7] S. Sessa, "On a Weak Commutativity Condition in Fixed Point Considerations," Publications de l'Institut Mathématique (Beograd), Vol. 34, No. 46, 1982, pp. 149-153.

[8] H. Bouhadjera and C. Godet-Thobie, "Common Fixed Theorems for Pairs of Subcompatible Maps," 2009.

[9] D. Gopal and M. Imdad, "Some New Common Fixed Point Theorems in Fuzzy Metric Spaces," Annali dell' Università di Ferrara, Vol. 57, No. 2, 2011, pp. 303-316. doi:10.1007/s11565-011-0126-4

[10] S. Manro, H. Bouharjera and S. Singh, "A Common Fixed Point Theorem in Intuitionistic Fuzzy Metric Space by Using Sub-Compatible Maps," International Journal of Contemporary Mathematical Sciences, Vol. 55, No. 55, 2010, pp. 2699-2707.

[11] G. Deschrijver and E. E. Kerre, "On the Relationship between Some Extensions of Fuzzy Set Theory," Fuzzy Sets and Systems, Vol. 133, No. 2, 2003, pp. 227-235. doi:10.1016/S0165-0114(02)00127-6

[12] G. Deschrijver, C. Cornelis and E. E. Kerre, "On the Representation of Intuitionistic Fuzzy T-Norm and T-Conorm," The IEEE Transactions on Fuzzy Systems, Vol. 12, No. 2, 2004, pp. 45-61. doi:10.1109/TFUZZ.2003.822678

[13] M. Tanveer, M. Imdad, D. Gopal and D. Kumar, "Common Fixed Point Theorem in Modified Intuitionistic Fuzzy Metric Spaces with Common Property (E.A.)," Fixed Point Theory and Applications, Vol. 36, 2012. doi:10.1186/1687-1812-2012-36

[14] D. Turkoglu, C. Alaca, Y. J. Cho and C. Yildiz, "Common Fixed Point Theorems in Intuitionistic Fuzzy Metric Spaces," Journal of Applied Mathematics and Computing, Vol. 22, No. 1-2, 2006, pp. 411-424. doi:10.1007/BF02896489

[15] C. Alaca, "A Common Fixed Point Theorem for Weak Compatible Mappings in Intuitionistic Fuzzy Metric Spaces," International Journal of Pure and Applied Mathematics, Vol. 32, No. 4, 2006, pp. 25-36.

[16] S. Manro, S. Kumar and S. S. Bhatia, "Common Fixed Point Theorems in Intuitionistic Fuzzy Metric Spaces Using Occasionally Weakly Compatible Maps," The Journal of Mathematics and Computer Science, Vol. 2, No. 2, 2012, pp. 73-81.

[17] R. P. Pant, "Common Fixed Foints of Four Mappings," Bulletin of Calcutta Mathematical Society, Vol. 90, No. 4, 1998, pp. 281-286. 